Maria Inês Couto de Oliveira'

Marcos Augusto Bastos Dias"

Cynthia B Cunha"'I

Maria do Carmo Leal' ${ }^{\mathrm{IV}}$
Departamento de Epidemiologia e Bioestatística. Instituto de Saúde da Comunidade. Universidade Federal Fluminense. Niterói, RJ, Brasil

Instituto Fernandes Figueira. Fundação Oswaldo Cruz (Fiocruz). Rio de Janeiro, RJ, Brasil

III Departamento de Informações em Saúde. Centro de Informação Científica e Tecnológica. Fiocruz. Rio de Janeiro, RJ, Brasil

Vepartamento de Epidemiologia e Métodos Quantitativos em Saúde. Escola Nacional de Saúde Pública. Fiocruz. Rio de Janeiro, RJ, Brasil

Correspondência | Correspondence: Maria Inês Couto de Oliveira

Departamento de Epidemiologia e Bioestatística

Instituto de Saúde da Comunidade

Universidade Federal Fluminense

R. Marquês do Paraná 303/3o andar

Prédio Anexo

24030-210 Niterói, RJ, Brasil

E-mail: marinesco@superig.com.br

\section{Qualidade da assistência ao trabalho de parto pelo Sistema Único de Saúde, Rio de Janeiro (RJ), 1999-2001}

\author{
Quality assessment of labor \\ care provided in the Unified \\ Health System in Rio de Janeiro, \\ Southeastern Brazil, 1999-2001
}

RESUMO

OBJETIVO: A qualidade da assistência ao trabalho de parto tem sido reconhecida na prevenção de complicações obstétricas que podem levar à morbi-mortalidade materna, perinatal e neonatal. O objetivo do estudo foi analisar a qualidade da assistência ao trabalho de parto segundo o risco gestacional e tipo de prestador.

MÉTODOS: Estudo transversal de observação da assistência ao trabalho de parto de 574 mulheres, selecionadas por amostra estratificada em 20 maternidades do Sistema Único de Saúde do Rio de Janeiro (RJ), entre 1999 e 2001. A qualidade da assistência foi analisada segundo o risco gestacional e o tipo de prestador. Utilizaram-se procedimentos estatísticos de análise de variância e de diferença de proporções.

RESULTADOS: Do total da amostra, 29,6\% das gestantes foram classificadas como de risco. Apesar da hipertensão ser a causa mais importante de morte materna no Brasil, a pressão arterial não foi aferida em 71,6\% das gestantes durante a observação no pré-parto. Em média foram feitas cinco aferições por parturiente, sendo o menor número nos hospitais conveniados privados (média de 2,9). Quanto à humanização da assistência, observou-se que apenas $21,4 \%$ das parturientes tiveram a presença de acompanhante no pré-parto, $75,7 \%$ foram submetidas à hidratação venosa e $24,3 \%$ à amniotomia. $\mathrm{O}$ único tipo de cuidado que variou segundo o risco obstétrico foi a freqüência da aferição da pressão arterial, em que as gestantes de risco foram monitoradas o dobro de vezes em relação às demais (média de $0,36 \times 0,18$ aferições $/ \mathrm{h}$ respectivamente, $\mathrm{p}=0,006)$.

CONCLUSÕES: De modo geral, as gestantes de baixo risco são submetidas a intervenções desnecessárias e as de alto risco não recebem cuidado adequado. Como conseqüência, os resultados perinatais são desfavoráveis e as taxas de cesariana e de mortalidade materna são incompatíveis com os investimentos e a tecnologia disponível.

DESCRITORES: Trabalho de Parto. Assistência Perinatal. Serviços de Saúde Materno-Infantil. Qualidade da Assistência à Saúde. Estudos Transversais. 


\begin{abstract}
OBJECTIVE: Quality of labor care has been recognized as a major factor for prevention of obstetric complications which can lead to maternal, perinatal and neonatal morbidity and mortality. The objective of the study was to assess quality of labor care by gestational risk and type of health provider.
\end{abstract}

METHODS: Observational, cross-sectional study of labor care provided to 574 pregnant women. Stratified sampling in 20 Unified Health System maternity hospitals in Rio de Janeiro, Brazil, was carried out between 1999 and 2001. Quality of labor care was assessed by gestational risk and type of health provider. Statistical analyses consisted of variance analysis and the analysis of difference between proportions.

RESULTS: Of all women studied, $29.6 \%$ were classified at obstetric risk. Although hypertension is the main cause of maternal death in Brazil, $71.6 \%$ did not have their prelabor blood pressure measured. Five measures were taken on average per parturient and the lowest rate was found in privately insured hospitals (average of 2.9). As to humanized health care, only $21.4 \%$ of the parturients had an accompanying person on their side during labor, $75.7 \%$ were submitted to intravenous hydration and $24.3 \%$ to amniotomy. The single care-related factor that varied by obstetric risk was frequency of blood pressure measures: high-risk parturients had their blood pressure measured twice as much as those low-risk women (mean 0.36 vs. 0.18 measures $/ \mathrm{h}, \mathrm{p}=0.006$ ).

CONCLUSIONS: In general, low-risk parturients were submitted to unnecessary interventions while high-risk women did not receive adequate care. As a result, there are poor perinatal outcomes, high cesarean rates and high maternal mortality rates, which do not reflect health care investments and technology available.

DESCRIPTORS: Labor, Obstetric. Perinatal Care. Maternal-Child Health Services. Quality of Health Care. Cross-Sectional Studies.

\title{
INTRODUÇÃO
}

A qualidade da assistência à mulher durante o trabalho de parto tem sido reconhecida na prevenção de complicações obstétricas que podem levar a um aumento da morbi-mortalidade materna, perinatal e neonatal.,6,9 No município do Rio de Janeiro (RJ) a mortalidade perinatal tem apresentado queda nas últimas décadas, mas permanece em níveis elevados (17,9\%o em 2001 e $16,4 \%$ em 2004) quando comparada à dos países desenvolvidos. A mortalidade perinatal peso-específica (peso $>2500 \mathrm{~g}$ ) apresentou taxa de 5,3\% em 2001 e de $4,3 \%$ em 2004 , correspondendo a mais de $61 \%$ do total de óbitos perinatais. ${ }^{\mathrm{a}}$

Embora, segundo a Organização Mundial da Saúde (OMS), entre $70 \%$ e $80 \%$ das gestantes possam ser consideradas de baixo risco no início do trabalho de parto, elas devem ser acompanhadas por profissional capacitado,${ }^{14}$ independentemente do risco gestacional. O papel do profissional de saúde na assistência ao parto é garantir o apoio à mulher e sua família, monitorar o bem-estar do binômio mãe-bebê, ${ }^{3}$ realizar as intervenções necessárias e quando indicado, referi-la para um nível mais complexo de cuidado. ${ }^{15}$

O acompanhamento adequado deste período incluir a observação de: temperatura axilar, pressão arterial, atividade uterina, evolução da dilatação do colo uterino pelo toque vaginal e batimentos cardíacos fetais, além da avaliação do bem-estar emocional da mulher, entre outros. ${ }^{15, \mathrm{~b}} \mathrm{O}$ monitoramento desses parâmetros deve ser feito regularmente e registrado em um partograma, ${ }^{13}$ permitindo à equipe avaliar a evolução do trabalho de parto e a necessidade de intervenção.

a Ministério da Saúde. Sistema de Informação sobre Mortalidade. Brasília; 1975 [citado 2007 set 15]. Disponível em: http://w3.datasus.gov. br/datasus/datasus.php?area=359A1B378C5D0E0F359G22H011Jd5L25M0N\&VInclude=../site/infsaude.php\&VObj=http://tabnet.datasus.gov. $\mathrm{br} / \mathrm{cgi} / \mathrm{deftohtm.exe?sim/cnv/obt}$

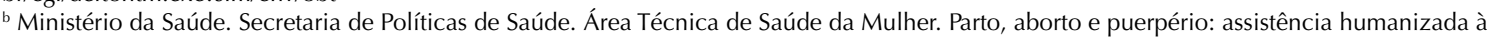
mulher. Brasília; 2001. 
Nas maternidades do Sistema Único de Saúde (SUS) a parturiente pode ser acompanhada por diferentes profissionais de uma mesma equipe de plantão ou mesmo por mais de uma equipe, dependendo do tempo entre sua internação e o nascimento do bebê. $\mathrm{O}$ registro em prontuário possibilita o monitoramento da assistência quando há mudança de equipe de plantão. Estudos anteriores sobre a qualidade da assistência ao trabalho de parto baseados em consulta a prontuário apontam a precariedade da atenção prestada ao trabalho de parto. ${ }^{4}$

A qualidade da assistência ao trabalho de parto e o sucesso do seu resultado inicia-se no momento da internação hospitalar. Idealmente, mulheres com gestações não complicadas e que não estão na fase ativa do trabalho de parto ( $\leq 5 \mathrm{~cm}$ de dilatação) não deveriam ser admitidas na área de pré-parto, pois correm o risco de serem submetidas a intervenções desnecessárias. ${ }^{71}$ Por outro lado, gestantes com riscos previamente detectados devem ser monitoradas mais freqüentemente.

Desde os anos 1980 tem sido apontada a necessidade de mudanças nas rotinas de assistência ao trabalho de parto ${ }^{9,14,15}$ para garantir melhores resultados perinatais, bem como a humanização da experiência do parto e nascimento vivenciadas pela mulher e sua família. ${ }^{\mathrm{b}, \mathrm{c}}$ A livre movimentação é uma das recomendações de bem-estar da mulher durante o trabalho de parto, pois a não-permanência em decúbito dorsal resulta em percepção de dor menos intensa, e conseqüentemente, menor necessidade de analgesia e de intervenções para correção da dinâmica uterina. ${ }^{15}$ A possibilidade de ter um acompanhante de sua escolha também favorece a evolução do trabalho de parto e diminui a necessidade do uso de analgésicos e de outras intervenções. ${ }^{1}$

O presente estudo teve como objetivo analisar a qualidade da assistência ao trabalho de parto segundo o risco gestacional da mulher e o tipo de prestador do hospital.

\section{MÉTODOS}

O estudo faz parte de pesquisa ampla, denominada "Estudo da Morbi-Mortalidade e da Atenção Peri e Neonatal no Município do Rio de Janeiro, 1999-2001" "5 sobre os procedimentos realizados no pré-parto nas instituições que pertenciam ao SUS. O estudo realizado foi transversal ${ }^{12} \mathrm{em}$ amostra estratificada proporcional de estabelecimentos do SUS, agrupados segundo a proporção de baixo peso ao nascer (BPN) em dois estratos: 1) municipais e federais; 2) estaduais, militares, filantrópicos e privados conveniados com o SUS.
O primeiro estrato amostral concentrou basicamente serviços de referência para gestações de alto risco e o segundo estrato abrangeu em geral maternidades de baixa complexidade, por não disporem de unidade de terapia intensiva (UTI)/unidade intensiva (UI) neonatal. Nos hospitais do SUS escolheu-se, ao acaso, uma a cada dez mulheres da amostra da pesquisa ampla, gerando uma sub-amostra de 574 ( $10 \%$ do total) parturientes. Foram consideradas perdas as duplicidades de registro de uma mesma parturiente em diferentes turnos de observação no pré-parto (não sendo considerado o segundo registro).

A observação foi conduzida por acadêmicos bolsistas de medicina e enfermagem, previamente treinados, com supervisão de campo. Esta observação durava quatro horas ou menos, caso a parturiente fosse encaminhada para a sala de parto ou centro cirúrgico. Assim, nem todas as parturientes tiveram um tempo de observação igual, variando em função da evolução do trabalho de parto de cada uma. $\mathrm{O}$ acompanhamento contemplou os períodos diurno e noturno de todos os dias da semana, inclusive os finais de semana e feriados.

Os dados foram coletados de três fontes: a consulta aos prontuários de dados da admissão, observação direta no pré-parto dos procedimentos de monitoramento do trabalho de parto e entrevista com parturientes.

Foi avaliado o uso do partograma durante o trabalho de parto. O partograma é um instrumento que possibilita diagnosticar alterações e indicar a tomada de condutas apropriadas para a correção destes desvios, ajudando ainda a evitar intervenções desnecessárias. A marcação adequada das linhas de alerta e de ação neste registro são fundamentais para a utilização correta deste instrumento. ${ }^{13}$

Os dados secundários do prontuário sobre admissão da gestante, data e horário de admissão, dia da semana e condições iniciais das gestantes foram transcritos em formulários padronizados.

Durante a observação do trabalho de parto, foram anotadas em formulários estruturados as seguintes aferições: medição de pressão arterial (PA), ausculta da freqüência cardíaca-fetal (FCF), avaliação pelo toque ginecológico da dilatação cervical (DC) e avaliação da dinâmica uterina (DU). Observou-se também realização de procedimentos de amniotomia, hidratação venosa, se houve deambulação, presença de acompanhante e encaminhamento a parto vaginal ou cesáreo.

As mães foram entrevistadas acerca de características maternas, condições obstétricas ou intercorrências clínicas.

a Carvalho ML. Mortalidade neonatal e aspectos da qualidade da atenção à saúde na Região Metropolitana do Rio de Janeiro em 1986/87 [dissertação de mestrado]. Rio de Janeiro: Escola Nacional de Saúde Pública da FIOCRUZ; 1993.

${ }^{\mathrm{b}}$ Rede pela Humanização do Parto e Nascimento. Carta de Campinas. Campinas;1993 [citado 2006 set 10]. Disponível em: http://www.rehuna.org.br/images/stories/documentos/carta\%20de\%20campinas.doc

c Rede pela Humanização do Parto e Nascimento. Carta de Fortaleza. Fortaleza; 2000 [citado 2006 set 10]. Disponível em:

http://www.rehuna.org.br/images/stories/ documentos/carta\%20de\%20fortaleza.doc 
As gestantes em risco obstétrico foram classificadas segundo a presença das seguintes condições, doenças obstétricas ou intercorrências clínicas: Rh negativo, hemorragia durante a gestação, diabetes pré-existente, diabetes gestacional, trabalho de parto prematuro, hipertensão arterial sistêmica pré-existente, hipertensão gestacional, pré-eclampsia, cardiopatias e doenças infecciosas (rubéola, sífilis, HIV e toxoplasmose).

A análise estatística consistiu de análise de variância e do teste qui-quadrado de Pearson. Na ocorrência de caselas com contagem esperada menor que 5 foi realizado o teste exato de Fisher. ${ }^{8}$ Os programas estatísticos utilizados foram o SPSS 10.0 e R 2.5.1. Para avaliação da adequação do acesso ao atendimento pré-natal utilizou-se o escore de Kotelchuck, ${ }^{6}$ que utiliza como parâmetros a época do início do pré-natal e a quantidade de consultas realizadas.

O estudo foi aprovado pelo Comitê de Ética para Pesquisa da Escola Nacional de Saúde Pública da Fiocruz. As parturientes assinaram um termo de consentimento à sua participação no estudo, tendo o anonimato sido assegurado.

\section{RESULTADOS}

As gestantes caracterizaram-se como de cor branca $(42,7 \%)$, parda $(32,2 \%)$, negra $(22,6 \%)$ e amarela $(2,5 \%)$. A clientela revelou-se como de baixo nível socioeconômico, com apenas $1,7 \%$ tendo completado o ensino superior, $17,8 \%$ o ensino médio e $28,2 \%$ o ensino fundamental. Verificou-se alta concentração de primíparas $(53,8 \%)$ (Tabela 1$)$.

Quando indagadas sobre problemas na gestação atual, $41,1 \%$ referiram anemia, 23,5\% infecção urinária, $0,4 \%$ tuberculose e $0,2 \%$ reportou ter usado droga ilícita durante a gravidez. Foram classificadas como parturientes de risco 29,6\%: 6,7\% referiram ter tido hemorragia durante a gravidez, $6,5 \%$ hipertensão pré-existente, $4,6 \%$ quadro anterior de pré-eclâmpsia, 2,4\% ter apresentado sífilis, $1,0 \%$ cardiopatia, $0,8 \%$ toxoplasmose, $0,8 \%$ diabetes gestacional, $0,6 \%$ ser Rh negativa, $0,4 \%$ ser HIV positiva, $0,4 \%$ diabetes pré-existente, $0,2 \%$ rubéola. Nenhuma relatou ser portadora de hepatite B e 10,3\% estavam em trabalho de parto prematuro.

A maioria das parturientes $(95,8 \%)$ havia feito algum tipo de acompanhamento pré-natal, das quais 58,4\% fizeram sete ou mais consultas, $33,9 \%$ de quatro a seis e 7,7\% entre zero e três consultas. Quase dois terços $(62,8 \%)$ iniciaram o pré-natal no primeiro trimestre da gestação, 30,0\% no segundo, 3,0\% no terceiro, e 4,2\% não fizeram pré-natal. Das gestantes, $89,4 \%$ fizeram acompanhamento pré-natal até o fim da gestação e $6,4 \%$ interromperam o acompanhamento antes do final.
Analisando-se o acompanhamento pré-natal realizado, $7,4 \%$ das gestantes de risco não fizeram pré-natal, contra $3,1 \%$ entre as gestantes de baixo risco. Do total das gestantes de risco, o acompanhamento de $38,3 \%$ foi inadequado (contra 30,0\% no baixo risco), de 33,6\% foi intermediário (contra $42,6 \%$ no baixo risco), de $14,8 \%$ foi adequado (contra $22,0 \%$ no baixo risco) e de $6,0 \%$ foi mais que adequado (contra $2,3 \%$ no baixo risco) $(\mathrm{p}=0,004)$. O pré-natal em $30,2 \%$ dos casos foi realizado no hospital onde se deu o parto.

Mais da metade das parturientes $(52,1 \%)$ foi assistida por maternidade municipal, $11,7 \%$ por estabelecimento filantrópico, 10,6\% por hospital contratado, 9,2\% por hospital militar, $8,7 \%$ por hospital estadual e $7,7 \%$ por hospital federal ou universitário. Quanto à complexidade das maternidades, $55,7 \%$ das parturientes foram internadas em maternidades com UTI/UI neonatal e as demais em maternidades sem UTI/UI neonatal. Foram admitidas em dias úteis $74,2 \%$ das gestantes e $25,8 \%$ em fins de semana, $58,4 \%$ das observações foram realizadas no turno diurno e $41,6 \%$ no noturno.

As condições iniciais das parturientes segundo prontuário estão apresentadas na Tabela 2. Verificou-se que $25,4 \%$ não teve sua PA aferida ou registrada na admissão. Entre as demais, 24,3\% apresentaram pressão

Tabela 1. Características das parturientes assistidas no préparto em maternidades do Sistema Único de Saúde. Rio de Janeiro, RJ, 1999-2001.

\begin{tabular}{|c|c|c|}
\hline Característica da parturiente & $\mathrm{n}$ & $\%$ \\
\hline \multicolumn{3}{|l|}{ Faixa etária (anos) } \\
\hline Até 19 & 141 & 26,6 \\
\hline $20-34$ & 343 & 64,7 \\
\hline 35 e mais & 46 & 8,7 \\
\hline \multicolumn{3}{|l|}{ Escolaridade } \\
\hline $\begin{array}{l}\text { Analfabeta à } 3^{\text {a }} \text { série do ensino } \\
\text { fundamental }\end{array}$ & 63 & 11,9 \\
\hline $4^{\mathrm{a}}$ à $7^{\mathrm{a}}$ série do ensino fundamental & 214 & 40,4 \\
\hline Ensino fundamental completo & 149 & 28,2 \\
\hline Ensino médio completo & 94 & 17,8 \\
\hline Ensino superior completo & 9 & 1,7 \\
\hline \multicolumn{3}{|l|}{ Cor da pele } \\
\hline Branca & 225 & 42,7 \\
\hline Parda & 170 & 32,2 \\
\hline Negra & 119 & 22,6 \\
\hline Amarela & 13 & 2,5 \\
\hline \multicolumn{3}{|l|}{ Paridade } \\
\hline Primigesta & 284 & 53,8 \\
\hline $2^{\mathrm{a}}$ ou $3^{\mathrm{a}}$ gestação & 180 & 34,1 \\
\hline $4^{\mathrm{a}}$ gestação e mais & 64 & 12,1 \\
\hline
\end{tabular}


Tabela 2. Condições iniciais das parturientes na admissão a maternidades do Sistema Único de Saúde. Rio de Janeiro, RJ, 1999-2001.

\begin{tabular}{lcr}
\hline Condição inicial da parturiente & $\mathrm{n}$ & $\%$ \\
\hline Não aferida ou não registrada & & \\
$\quad$ Pressão arterial & 146 & 25,4 \\
$\quad$ Freqüência cardíaca fetal & 110 & 19,2 \\
Dilatação cervical & 24 & 4,2 \\
$\quad$ Dinâmica uterina & 154 & 26,8 \\
Temperatura & 527 & 91,8 \\
Pressão arterial (mmHg) & & \\
PA sistólica $\geq 140$ e/ou PA diastólica $\geq 90$ & 104 & 24,3 \\
Freqüência cardíaca fetal & & \\
$\quad<120$ bpm ou >160 bpm & 5 & 1,1 \\
Dilatação cervical & & \\
0-4 cm & 329 & 64,6 \\
5 -10 cm & 180 & 35,4 \\
\hline
\end{tabular}

alta (PA sistólica $\geq 140 \mathrm{mmHg}$ e/ou PA diastólica $\geq 90$ $\mathrm{mmHg}$ ). Na admissão de 19,2\% das gestantes não foram aferidos ou registrados os batimentos cardíacos fetais e, dentre as que foram, em $1,1 \%$ dos casos foi observada bradicardia $(<120 \mathrm{bpm})$ ou taquicardia $(>160 \mathrm{bpm})$.

A avaliação da DC pelo exame vaginal foi a medida mais verificada no momento da admissão, não havendo registro para $4,2 \%$ dos casos. No momento da admissão, $64,6 \%$ das parturientes tinham o colo dilatado até $4 \mathrm{~cm}$ e $35,4 \%$ apresentavam DC entre 5 e $10 \mathrm{~cm}$. Não houve diferença significativa na DC à admissão entre as gestantes de baixo ou alto risco. A dinâmica uterina (número de contrações em cada dez minutos) de 26,8\% das parturientes não foi verificada na admissão. A temperatura axilar não pôde ser avaliada, pois não havia registro para $91,8 \%$ dos casos.
O partograma foi utilizado no acompanhamento do trabalho de parto da maior parte (92\%) das parturientes, com uma variação de $65 \%$ nos hospitais militares até $98 \%$ nos hospitais estaduais e filantrópicos. No entanto, foram observados sub-registro e registro irregular das diferentes avaliações maternas e fetais.

Na Tabela 3 observa-se que uso da hidratação venosa foi prática rotineira na assistência ao trabalho de parto $(75,7 \%)$, mais observada nos estabelecimentos filantrópicos $(86,6 \%)$ e militares $(84,9 \%)$. Não houve diferença significativa na sua utilização entre as gestantes de baixo $(74,9 \%)$ e alto risco $(76,4 \%)$. Das parturientes, $61,3 \%$ fizeram uso de ocitocina.

Quanto a aspectos da humanização da assistência ao pré-parto (Tabela 3), 21,4\% tiveram a presença de um acompanhante no pré-parto. A presença de acompanhante foi semelhante entre as diversas faixas etárias: $22,7 \%$ entre as adolescentes, $21,3 \%$ entre as parturientes de 20 a 34 anos e $21,7 \%$ entre aquelas com 35 anos ou mais. Dois terços dos acompanhantes $(64,2 \%)$ eram do sexo feminino e um terço $(35,8 \%)$ do sexo masculino. Os acompanhantes mais comuns eram a mãe ou pai da parturiente ( $38,7 \%$ dos casos), o pai do bebê/companheiro (37,0\%), outros parentes (17,6\%) e amigas ou outro $(5,0 \%)$. A presença de acompanhante foi mais freqüente nas maternidades militares $(58,5 \%) \mathrm{e}$ inexistente $(0 \%)$ nos hospitais privados contratados.

Apenas $11,4 \%$ das parturientes foi estimulada a deambular e em $11,0 \%$ dos casos a deambulação foi reprimida; em $19,0 \%$ a deambulação foi permitida e em $58,6 \%$ não foi possível determinar. $\mathrm{O}$ estímulo à deambulação foi mais freqüente nos hospitais filantrópicos $(19,7 \%) \mathrm{e}$ estaduais $(18,8 \%)$ e a deambulação foi mais reprimida nos estabelecimentos privados contratados $(26,8 \%)$ e militares $(18,9 \%)$. Cerca de um quarto das parturientes $(24,3 \%)$ foi submetida à amniotomia, variando de $42,4 \%$ nos hospitais filantrópicos a $10,9 \%$ nos estaduais.

Tabela 3. Procedimentos relacionados à humanização da assistência ao pré-parto por tipo de prestador em maternidades do Sistema Único de Saúde. Rio de Janeiro, RJ, 1999-2001.

\begin{tabular}{|c|c|c|c|c|c|c|c|c|c|c|c|c|c|c|}
\hline \multirow{3}{*}{ Procedimento } & \multicolumn{14}{|c|}{ Tipo de prestador } \\
\hline & \multicolumn{2}{|c|}{ Municipal } & \multicolumn{2}{|c|}{ Federal } & \multicolumn{2}{|c|}{ Estadual } & \multicolumn{2}{|c|}{ Militar } & \multicolumn{2}{|c|}{ Filantrópico } & \multicolumn{2}{|c|}{ Contratado } & \multicolumn{2}{|c|}{ Total } \\
\hline & $\mathrm{n}$ & $\%$ & $\mathrm{n}$ & $\%$ & $\mathrm{n}$ & $\%$ & $\mathrm{n}$ & $\%$ & $\mathrm{n}$ & $\%$ & $\mathrm{n}$ & $\%$ & $\mathrm{n}$ & $\%$ \\
\hline $\begin{array}{l}\text { Presença de } \\
\text { acompanhante }\end{array}$ & $82 / 299$ & 27,4 & $8 / 44$ & 18,2 & $1 / 50$ & 2,0 & $31 / 53$ & 58,5 & $1 / 67$ & 1,5 & $0 / 61$ & 0,0 & $123 / 574$ & 21,4 \\
\hline $\begin{array}{l}\text { Deambulação } \\
\text { estimulada }\end{array}$ & $33 / 87$ & 11,2 & $2 / 15$ & 4,5 & $9 / 34$ & 18,8 & $7 / 28$ & 13,2 & $13 / 41$ & 19,7 & $0 / 28$ & 0,0 & $64 / 233$ & 11,4 \\
\hline $\begin{array}{l}\text { Deambulação } \\
\text { reprimida }\end{array}$ & $24 / 87$ & 8,1 & $3 / 15$ & 6,8 & $2 / 34$ & 4,2 & $10 / 28$ & 18,9 & $8 / 41$ & 12,1 & $15 / 28$ & 26,8 & $62 / 233$ & 11,0 \\
\hline Amniotomia & $58 / 286$ & 20,3 & $14 / 42$ & 33,3 & $5 / 46$ & 10,9 & $15 / 51$ & 29,4 & $28 / 66$ & 42,4 & $13 / 56$ & 23,2 & $133 / 547$ & 24,3 \\
\hline $\begin{array}{l}\text { Hidratação } \\
\text { venosa }\end{array}$ & $230 / 299$ & 76,9 & $28 / 44$ & 63,6 & $35 / 50$ & 70,0 & $45 / 53$ & 84,9 & $58 / 67$ & 86,6 & $36 / 58$ & 62,1 & $432 / 571$ & 75,7 \\
\hline $\begin{array}{l}\text { Média de } \\
\text { verificações } \\
\text { por mulher }\end{array}$ & 285 & 5,1 & 40 & 5,1 & 48 & 4,6 & 50 & 5,2 & 59 & 6,8 & 59 & 2,9 & 541 & 5,0 \\
\hline
\end{tabular}


Tabela 4. Procedimentos de assistência ao trabalho de parto e desfechos maternos e neonatais segundo o risco gestacional em maternidades do Sistema Único de Saúde. Rio de Janeiro, RJ, 1999-2001.

\begin{tabular}{lccccc}
\hline \multirow{2}{*}{ Procedimento e desfecho do trabalho de parto } & \multicolumn{2}{c}{ Baixo risco } & \multicolumn{2}{c}{ Risco } & $\mathrm{p}$ \\
& \% ou média & $\mathrm{n}$ & \% ou média & $\mathrm{n}$ & 0,261 \\
\hline Média de verificações/h & 2,83 & 349 & 3,15 & 148 & 0,006 \\
Média de verificações de PA/h & 0,18 & 363 & 0,36 & 155 & 0,607 \\
Média de toques/h & 1,26 & 368 & 1,33 & 156 & 0,819 \\
Média de auscultas FCF/h & 1,05 & 362 & 1,08 & 154 & 0,215 \\
Média de verificações de dinâmica uterina/h & 0,41 & 366 & 0,49 & 153 & 0,001 \\
Intercorrências com a gestante durante o parto & $5,9 \%$ & 22 & $12,1 \%$ & 19 & 0,074 \\
Parto cesáreo & $20,6 \%$ & 77 & $26,5 \%$ & 41 & 0,098 \\
Baixo peso ao nascer & $4,9 \%$ & 18 & $19,0 \%$ & 29 & 0,059 \\
APGAR 5 $5^{\circ}$ minuto $<7$ & $2,3 \%$ & 8 & $9,8 \%$ & 15 & 0,001 \\
Recém-nascido encaminhado para UTI/UI & $5,7 \%$ & 21 & $17,8 \%$ & 28 & \\
\hline
\end{tabular}

PA: pressão arterial

FCF: freqüência cardíaca-fetal

O tempo médio de observação das parturientes foi de 146 minutos, variando de $<60 \min (17,8 \%)$, a $\geq 60$ $\min \mathrm{a} \leq 180 \mathrm{~min}(46,5 \%)$, até $>180 \mathrm{~min} \mathrm{a}<300 \mathrm{~min}$ $(35,7 \%)$. Durante o período de observação do pré-parto, em média foram realizadas cinco verificações por parturiente, sendo 2,1 de toque, 1,7 de ausculta de FCF, 0,9 de avaliação da DU e 0,4 de medida da PA. Durante este acompanhamento, $71,6 \%$ das mulheres não tiveram sua PA aferida, 50,9\% não tiveram a DU aferida e $11,9 \%$ não tiveram a DC verificada. Em todas foi feita ao menos uma verificação da FCF. O maior número de verificações ocorreu nos hospitais filantrópicos (média de 6,8 ) e o menor número nos contratados (média de 2,9). Não houve diferença estatisticamente significativa no número de verificações realizadas nas parturientes segundo o dia ou horário de observação.

A verificação realizada com maior freqüência foi o toque (41,8\% das aferições), seguido pela FCF $(33,4 \%)$, DU $(17,0 \%)$ e PA $(7,7 \%)$, num total de 2.933 verificações realizadas no conjunto das 574 parturientes. O profissional que mais executou o monitoramento da parturiente foi o médico (69,0\% das verificações), seguido do estudante de medicina (16,3\%), do enfermeiro (12,2\%) e de auxiliar de enfermagem ou outro $(2,5 \%)$.

A maior parte das gestantes de risco foi assistida por maternidades do estrato 1: $66,2 \%$ versus $49,1 \%$ pelo estrato $2(\mathrm{p}<0,001)$. As maternidades municipais assistiram $64,3 \%$ das gestantes de risco, as militares $9,6 \%$, as federais/universitárias $8,9 \%$, as estaduais $8,9 \%$, as filantrópicas 7,0\% e as contratadas apenas assistiram às de baixo risco obstétrico. Não foi encontrada diferença significativa entre gestantes de risco e de baixo risco em relação à escolaridade $(\mathrm{p}=0,31)$, faixa etária $(\mathrm{p}=0,25)$ ou paridade $(\mathrm{p}=0,12)$.

$\mathrm{Na}$ Tabela 4, observa-se que não houve diferença significativa no número de verificações do bem-estar materno ou fetal realizadas por hora nos dois grupos $(3,15$ versus $2,83, p=0,261)$. O único tipo de verificação que variou entre os dois grupos foi a da PA, que apresentou uma média de 0,36 aferições entre as gestantes de risco e de 0,18 entre as demais $(p=0,006)$. As parturientes que foram admitidas precocemente na maternidade (com $4 \mathrm{~cm}$ ou menos de dilatação) foram as mais submetidas ao parto cesáreo $(27,1 \%$ versus $12,3 \%, \mathrm{p}<0,001)$. Apresentaram intercorrências durante o trabalho de parto $12,1 \%$ das gestantes de risco e 5,9\% daquelas de baixo risco $(\mathrm{p}=0,001)$.

Em relação ao bem-estar do recém-nascido, 15,2\% apresentaram índice de Apgar de $1^{\circ}$ minuto menor do que 7 e 4,6\% Apgar de $5^{\circ}$ minuto menor do que 7 . Após o parto, $17,8 \%$ dos recém-nascidos das gestantes de risco foram para a UI ou UTI em relação a 5,7\% dos bebês das gestantes de baixo risco $(p=0,001)$. Evoluíram para o óbito $0,9 \%$ dos recém-nascidos, todos filhos de gestantes de risco.

\section{DISCUSSÃO}

Apesar da utilização de observadores da rotina do préparto externos às instituições avaliadas, não consideramos que esta presença tenha interferido positivamente na rotina de monitoramento do trabalho de parto dos hospitais, devido à precariedade das práticas de assistência encontradas. No entanto, caso tenha ocorrido algum viés de cortesia, a realidade que o presente estudo revelou foi ainda conservadora.

Embora o Ministério da Saúde e a OMS ${ }^{15}$ recomendem que no momento da admissão seja feita uma avaliação do risco gestacional para definir o "potencial de nascimento" e as condutas a serem adotadas na condução do trabalho de parto, esta não foi a realidade observada nas maternidades do SUS do Município do Rio de Janeiro.

a Ministério da Saúde. Secretaria de Políticas de Saúde. Área Técnica de Saúde da Mulher. Parto, aborto e puerpério: assistência humanizada à mulher. Brasília; 2001. 
Em grande parte das vezes não foram verificados ou registrados elementos importantes na avaliação do risco gestacional e neonatal na admissão. Entre eles, a PA, pela alta prevalência da hipertensão como condição de risco; a freqüência cardíaca fetal, pela sua importância na prevenção da morbi-mortalidade neonatal; e a temperatura axilar, pela possibilidade de suspeição precoce de uma sepsis. ${ }^{15}$

A hipertensão arterial tem sido apontada como a principal causa de morte materna no Brasil. ${ }^{a}$ No relatório do Comitê de Prevenção e Controle da Morte Materna do Estado do Rio de Janeiro ${ }^{\mathrm{b}}$ esta patologia aparece como a mais importante causa de mortalidade materna entre 2001 e 2003, tendo sido responsável por mais de $25 \%$ dos óbitos. A avaliação da PA ficou abaixo do esperado, mesmo para gestantes de baixo risco: uma verificação pelo menos a cada quatro horas, ${ }^{2}$ embora as gestantes de maior risco tenham tido sua pressão arterial avaliada com freqüência duas vezes maior.

A pesquisa revelou ainda que cerca de dois terços das gestantes foram admitidas com dilatação do colo uterino menor ou igual a $4 \mathrm{~cm}$, evidenciando uma tendência generalizada de internação numa fase inicial do trabalho de parto, independentemente da presença de condição de risco associada à gestação.

A utilização do partograma observada nos serviços ainda não foi universal, nem realizada da forma recomendada para a melhoria efetiva dos resultados maternos e perinatais, comprometendo assim o acompanhamento do trabalho de parto. Este instrumento possibilita que uma das estratégias de redução de cesarianas, a condução ativa de distócias - aguardar pelo menos duas ou mais horas de contrações uterinas eficazes e regulares antes da indicação de cesárea - seja feita de forma adequada. ${ }^{10}$ Uma das principais recomendações para a utilização do partograma diz respeito ao diagnóstico correto do início da fase ativa do trabalho de parto para que o registro do acompanhamento seja feito apenas a partir deste momento, evitando diagnósticos e intervenções incorretas. Uma vez que grande parte das mulheres foi admitida precocemente, fica a preocupação de que o uso do mesmo possa não ter sido adequado. Nesse sentido, como em outros trabalhos, ${ }^{7}$ a proporção de parturientes admitidas precocemente foi significativamente maior a de cesarianas.

Outra conseqüência da admissão precoce é a realização de um maior número de toques vaginais, aumentando a possibilidade de uma infecção, ${ }^{16}$ além do desconforto para a mulher. Durante seu acompanhamento no pré-parto, as mulheres foram submetidas ao dobro de exames vaginais para avaliar a dilatação cervical do que o esperado (um exame a cada quatro horas durante a primeira fase do trabalho de parto).${ }^{13} \mathrm{~A}$ internação precoce acontece algumas vezes por questões de ordem social, como a pressão familiar pelas dificuldades com transporte, ou mesmo para assegurar uma vaga na maternidade. Nessas circunstâncias, em que os determinantes da admissão hospitalar não são de ordem médica, a melhor alternativa é evitar que a gestante seja colocada no pré-parto.

A adoção generalizada da hidratação venosa, independentemente da condição de risco materno, é contra-indicada nas gestantes de baixo risco. Além de dificultar a movimentação da parturiente, esse procedimento pode levar a hiperinsulinismo fetal se utilizada a infusão de glicose acima de $25 \mathrm{~g} .{ }^{15}$ Além dos problemas apontados, a colocação da venóclise facilita o uso de medicação para acelerar o trabalho de parto, muitas vezes sem indicação precisa, trazendo novos riscos para o binômio materno-fetal. A utilização de ocitocina para induzir ou acelerar o trabalho de parto em uma proporção tão elevada de parturientes também é preocupante, principalmente no País, com alta taxa de cesárea e acompanhamento do bem-estar materno ou fetal ainda precário. O uso desta medicação deve ser feito com vigilância do bem-estar materno e fetal, para evitar possíveis complicações, como a hipertonia uterina e o sofrimento fetal. As rotinas que levam à medicalização desnecessária da mulher durante o trabalho de parto devem ser repensadas pelos inconvenientes que podem trazer para a parturiente e para o feto, além de elevarem os custos hospitalares de um sistema de saúde com dificuldades de financiamento.

A ausculta dos batimentos cardíacos fetais, uma forma de diagnosticar o sofrimento fetal e evitar a asfixia perinatal e suas conseqüências, foi realizada com uma freqüência cerca de 50\% inferior à esperada (verificação a cada 15-30 minutos durante a primeira fase do trabalho de parto). ${ }^{15}$ Este monitoramento insuficiente é motivo de preocupação, em especial quando a utilização de ocitocina se deu com a alta freqüência observada. A investigação dos casos de natimortalidade e de asfixia perinatal pelas chefias dos serviços, e sua discussão com o corpo clínico da maternidade, são formas de sensibilizar os profissionais para um acompanhamento cuidadoso da freqüência cardíaca fetal.

Apesar das diversas vantagens proporcionadas pela presença de um acompanhante de escolha da mulher durante o trabalho de parto, menos de um quarto delas puderam usufruir deste direito. Este acompanhante esteve pouco presente, mesmo entre as gestantes adolescentes, cujo direito a esta prerrogativa está garantido desde $1990 .^{\mathrm{c}}$ A possibilidade de ter um acompanhante foi maior nas maternidades militares e nas municipais,

\footnotetext{
a Ministério da Saúde. Pacto nacional pela redução da mortalidade materna e neonatal. Brasília; 2004 [acesso 2006 jul 8]. Disponível em: http://dtr2002.saude.gov.br/proesf/14-Material//nfo/PactoAprovadoTripartite

b Secretaria de Estado de Saúde do Rio de Janeiro. Relatório do Comitê Estadual de Prevenção e Controle da Morte Materna e Perinatal. Rio de Janeiro; 2005 [citado 2006 jul 8]. Disponível em: http://www.saude.rj.gov.br/Acoes/RelatorioCMM2005

c Brasil. Lei 8.069, de 13 de julho de 1990. Dispõe sobre o Estatuto da Criança e do Adolescente e dá outras providências. Diário Oficial União. 16 jul 1990;Seção 1:013563.
} 
que na época já dispunham de uma política de humanização da assistência, porém ainda em níveis bastante inferiores aos desejados. Como a presença de acompanhante foi recentemente regulamentada pelo Ministério da Saúde ${ }^{a}$ para todas as parturientes, é fundamental que os serviços se estruturem para incluí-la em sua rotina de assistência. Da mesma forma, em relação à liberdade de movimentação, poucos serviços a estimulavam, e alguns a reprimiam, embora seja prática comprovadamente benéfica e recomendada. ${ }^{14}$ As maternidades privadas contratadas apresentavam os piores índices relativos à humanização da assistência, e nos dois anos posteriores à realização do presente estudo, estes serviços deixaram de prestar assistência à clientela do SUS.

\section{REFERÊNCIAS}

1. Bruggemann OM, Parpinelli MA, Osis MJD. Evidências sobre o suporte durante o trabalho de parto/parto: uma revisão da literatura. Cad Saude Publica. 2005;21(5):1316-27. DOI: 10.1590/S0102311X2005000500003

2. Cunningham FG, Gant NF, Leveno KJ, Gilstrap III LC, Hauth JC, Wenstrom KD. Williams obstetrics. 21.ed. New York: McGraw Hill; 2001. p.251-383.

3. Hofmeyr GJ. Evidence-based intrapartum care. Best Pract Res Clin Obstet Gynaecol. 2005;19(1):103-15.

4. Lansky S, França E, Leal MC. Mortalidade perinatal e evitabilidade: revisão de literatura. Rev Saude Publica. 2002;36(6):759-72. DOI: 10.1590/S003489102002000700017

5. Leal MC, Gama SG, Campos MR, Cavalini LT, Garbayo LS, Brasil CL, et al. Fatores associados à morbi-mortalidade perinatal em uma amostra de maternidades públicas e privadas do Município do Rio de Janeiro, 1999-2001. Cad Saude Publica. 2004;20(Supl 1):S20-33. DOI: 10.1590/S0102311X2004000700003

6. Leal MC, Gama SG, Ratto KM, Cunha CB. Uso do índice de Kotelchuck modificado na avaliação da assistência pré-natal e sua relação com as características maternas e o peso do recém-nascido no Município do Rio de Janeiro. Cad Saude Publica. 2004;20(Sup 1):S63-72. DOI: 10.1590/S0102$311 \times 2004000700007$

7. McNiven P, Williams JI, Hodnett E, Kaufman K, Hannah ME. An early labor assessment program: a
É necessário que as gestantes de baixo risco recebam suporte físico e emocional e seja respeitada a fisiologia do trabalho de parto. As mulheres com patologias intercorrentes na gestação precisam ter acesso a um atendimento criterioso, ${ }^{\mathrm{b}}$ com controle efetivo do seu quadro clínico, disponibilizando todos os recursos necessários para garantir o seu bem-estar e do feto.

Os gestores locais devem acompanhar de perto as condições de funcionamento das maternidades ${ }^{\mathrm{c}}$ e os resultados perinatais da assistência prestada. Devem discutir com a equipe de saúde formas de garantir melhores indicadores, ao mesmo tempo em que proporcionam às usuárias e suas famílias uma vivência positiva no momento do nascimento de uma criança. randomized, controlled trial. Birth. 1998;25(1):5-10. DOI: 10.1046/j.1523-536x.1998.00005.x

8. Mehta CR, Patel NR. Algorithm 643. FEXACT: a Fortran subroutine for Fisher's exact test on unordered $r^{*}$ C contingency tables. ACM Trans Math Softw. 1986;12(2):154-61. DOI: 10.1145/6497.214326

9. Rezende J, Montenegro CAB. Obstetrícia Fundamental. 10. ed. Rio de Janeiro: Guanabara Koogan; 2005. p.181-204.

10. Sanchez-Ramos L, Kaunitz AM, Peterson HB, Martinez-Schnell B, Thompson RJ. Reducing cesarean sections at a teaching hospital. Am J Obstet Gynecol. 1990;163(3):1081-7.

11. Schneck CA, Riesco MLG. Intervenções no parto de mulheres atendidas em um centro de parto normal intra-hospitalar. REME: Rev Min Enferm. 2006;10(3):240-6.

12. Szklo M, Javier-Nieto F. Epidemiology: beyond the basics. Maryland: Aspen Publishers; 2000.

13. World Health Organization. Preventing prolonged labour: a practical guide. The partograph. Geneva; 1993.

14. Appropriate technology for birth. Lancet. 1985;2(8452):436-7.

15. World Health Organization. Care in normal birth: a pratical guide. Maternal and Newborn Health / Safe Motherhood Unit. Family and Reproductive Health. Geneva; 1996.

16. World Health Organization. Postpartum care of the mother and newborn: a practical guide. Geneva; 1998.

Pesquisa realizada em parceria com a Secretaria Municipal de Saúde do Rio de Janeiro.

\footnotetext{
a Ministério da Saúde. Portaria $\mathrm{n}^{\circ} 2.418$, de 02 de dezembro de 2005. Regulamenta, em conformidade com o art. $1^{\circ}$ da Lei $\mathrm{n}^{\circ} 11.108$, de 7 de abril de 2005, a presença de acompanhante para mulheres em trabalho de parto, parto e pós-parto imediato nos hospitais públicos e conveniados com o Sistema Único de Saúde - SUS. Diário Oficial União. 06 dez 2005;Seção 1:32.

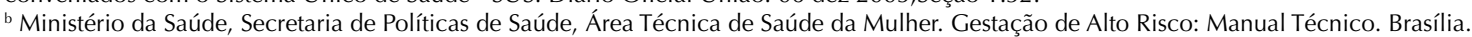
$3^{\mathrm{a}}$ ed. 2000.

c Rosas CF (coordenador). Avaliação dos serviços de assistência ao parto e ao neonato no Estado de São Paulo, 1997-1998. São Paulo:

Conselho Regional de Medicina do Estado de São Paulo; 1998.
} 